Methods We prospectively measured compliance with the guideline in a randomly selected subset. After doing the first cycle, we presented the results locally along with regular education of PICU medical and nursing staff. We compared the total number of blood tests requested and their costs per patient-day in three days-blocks pre-intervention (October 2019) and post-intervention (January 2020).

Results Prospective audit data showed compliance with the guideline in 8 of $24(33.3 \%)$ patient-days studied. The total number of tests increased from 179 to 191 (\% increase) in the post-intervention period. Patient-days had increased from 24 to 39 (\% increase) in the corresponding period.

The proportion of patients needing level 3 and 4 care 2 was higher in the post-intervention period (48.7\% vs. $41.6 \%)$. The average number of blood tests requested per patient-day decreased from 7.45 to 4.89 post-intervention, a $34.3 \%$ reduction. The average cost per patient-day decreased from $£ 36.6$ to $£ 24$ post-intervention, a $34.4 \%$ cost saving. The savings over 12 months would be $£ 51,458.4$ based on 4084 patient-days in 2019. There were no adverse events reported due to lack of blood results

Conclusions Blood testing rates can be safely reduced in critically ill children.

\section{USING GONAD SHIELDS IN PAEDIATRIC X-RAYS}

Fadi Maghrabia. UK

\subsection{6/bmjpo-2021-RCPCH.75}

Background The problem of inaccurate placement of gonad shields in children has been highlighted by several publications nationally and internationally. ${ }^{3}$

A written departmental protocol for the correct use of gonad protection, with regular audit, will:

- Avoid confusion over when and where gonad protection is required

- Help new staff and locum staff to adapt readily and easily to local policy

- Reduce gonad dose without significant loss of radiographic information

Objectives

- The aim of the study is to make sure that children's gonads are protected from the unnecessary harmful radiations caused by $\mathrm{x}$-rays that can affect them in the long term

- Gonad protection should be used according to the local policy in all cases. ${ }^{12}$ (The guidelines of the paediatric radiology department at BRI)

- Initial Hip/Pelvic x-ray doesn't require gonad shield to avoid obscuring the sacrum in females or symphysis pubis in males.

- Gonad shield should be used and positioned accurately in all subsequent images.

Methods

- PACS system was used to search for 2 views Hip/Pelvic X-ray in patients <16 years old at BRI hospital.

- Retrospective study (Audit: September 2017 - April 2018)

(Re-audit April 2017 - August 2018)

- Each image was assessed to determine the presence or absence of gonad protection and whether the protection was placed correctly or not.
Results

- The results were discussed at the radiological clinical governance meeting.

- The superintendent radiographer has sent a reminder e-mail to all the radiographers discussing the importance of not only using the gonad shield but also placing it correctly.

- Reminder posters to use the gonad shield were put in every $\mathrm{x}$-ray room.

- A course has been conducted for the new radiographers by a senior radiographer about when and how to use the gonad shield.

- The local guidelines have been reviewed and simplified to make it easier to follow.

- Gonad shields have been used in the correct position in $60 \%$ of patients after implementing the changes compared to $33 \%$ only in the first cycle.

Conclusions It was noticed that the percentage of the wellpositioned shields has improved dramatically from 33\% to $60 \%$ after implementing the previous changes.

\section{USING EXTUBATION CHECKLIST IN NICU TO IMPROVE THE SUCCESSFUL EXTUBATION RATE}

Fadi Maghrabia. UK

\subsection{6/bmjpo-2021-RCPCH.76}

Background Mechanical ventilation is a cornerstone of managing respiratory failure in preterm infants. Whilst its role to improve the survival rate has been proven, it can also result in unintended harm. ${ }^{12}$

It can increase the risk of developing bronchopulmonary dysplasia, sepsis, neurological injury, and retinopathy of prematurity. Because of that, clinicians aim to extubate preterm infants as early as possible. ${ }^{3} 4$

Given that approximately two-thirds of infants born before 29 weeks' gestation require intubation, an extubation checklist was implemented at the neonatal intensive care unit at Leeds General Infirmary hospital and St James hospital trying to improve the successful extubation rate.

Objectives To identify if using the extubation checklist has helped to reduce the rate of unsuccessful extubation in preterm infants in neonatal units at Leeds general infirmary hospital and St James hospital.

Methods

- A retrospective cohort study of extremely preterm infants ( $<27$ weeks gestational age) or ELBW infants (birth weight $<1000 \mathrm{~g}$ ) requiring mechanical ventilation during the period 01/01 - 30/06 in 2013 and 2018 (pre and postimplementation of the extubation list).

- Successful extubation is defined as not requiring reintubation for 72 hours post-extubation.

- Badger system was used to search for all patients who were admitted to LGI \& St James neonatal unites during the 2 periods of $01 / 01 / 2013-30 / 06 / 2013$ and $01 / 01 / 2018-30 / 06 /$ 2018.

- Notes for the identified patients were requested from the archive.

- Dates of extubation and re-intubation attempts were obtained from the notes

Inclusive criteria 
- $\quad<27$ weeks gestational age and/or birthweight $<1000$ grams

- Born between 01/01/2013 - 30/06/2013 and 01/01/2018-30/ 06/2018

- Required mechanical ventilation during the same period.

- The use of the extubation checklist in 2018 group

Results Amongst the pre-checklist cohort, 24 babies met the GA/BW criteria, with 3 sets of notes unavailable. Of these, 15 patients received mechanical ventilation. There were 27 extubation attempts, 19 of these were unsuccessful and 8 of them were successful (29.6\%).

In 2018, 31 babies met the GA/BW criteria, with 4 sets of notes unavailable, 17 patients needed ventilation, 30 extubation attempts using the extubation checklist, 16 of these were unsuccessful and 14 of them were successful (46.6\%)

Conclusions

- It was noted that the proportion of unsuccessful extubation attempts dropped by $17 \%$ (from $70.4 \%$ to $53.4 \%$ ) after implementing the extubation checklist.

- Extubation of extreme preterms is 1.6 more likely to be successful when extubation checklist is used.

\section{DO NEURAMINIDASE INHIBITORS IMPROVE OUTCOMES IN CRITICALLY UNWELL CHILDREN WITH INFLUENZA?}

Amedine Duret, Amedine Duret, Nikita Punjabi. UK

\subsection{6/bmjpo-2021-RCPCH.77}

Background Influenza is a common seasonal acute respiratory viral illness. Children, especially those with co-morbidities, are at risk of complications and ICU admission. No specific guidelines have been formulated about starting neuraminidase inhibitors (NAI) in critically ill children with influenza, but the Health Protection Agency and American Academy of Pediatrics have both stated that antiviral therapy should be initiated as soon as possible in this cohort.

Objectives We aimed to evaluate the evidence supporting the early initiation of NAI in critically ill children with influenza, by conducting a literature search to establish whether NAI improved survival and shortened intensive care admissions in children critically ill with influenza.

Methods We searched the literature for articles on the use of NAI treatment in critically unwell children or children in PICU diagnosed with influenza. We excluded articles with adult patients only, or with a mixture of adults and children where the results were not stratified by age. We also excluded articles with children in outpatient settings, or hospitalised on low-dependency units.

Results Out of 369 articles (Cochrane Library: 2, PUBMED: 328, NHS Evidence: 39), twelve studies published between 2010 and 2017 were included, seven of which were cohort studies (Level 3 evidence) and five case series (Level 4 evidence), with a total of over 7,000 critically ill children with influenza worldwide.

Six cohort studies compared mortality in children receiving NAI and children who did not: five of these demonstrated a trend towards decreased mortality with the use of NAI, and one showed no difference between treated and untreated groups. Only one study reached statistical significance, with $\mathrm{p}$ $=0.01$ for association of NAI treatment with survival. We noted that the two studies which reported on NAI-related adverse events reported none.
There is some evidence in our data that early NAI within 48 hours of symptom onset and/or admission has additional benefits compared to late NAI, although the largest cohort study did not demonstrate this effect.

The studies included here had limitations. Patient cohorts were heterogeneous, with some having had RT-PCR confirmed influenza and others just a clinical diagnosis. Few studies reported on concurrent treatment with antibiotics or steroids. Several studies stressed that patients receiving NAI were more likely to have co-morbidities and very severe influenza requiring mechanical ventilation at baseline, compared to patients who were not started on NAI.

Conclusions The current evidence on the use of NAI in critically ill children with influenza is weak (Level 3), but trends toward improved survival. The trend of improved survival is particularly salient if NAI treatment is initiated within 48 hours of symptom onset. This may mean starting oseltamivir on clinical suspicion of influenza, without waiting for a laboratory confirmation of the diagnosis, to avoid delay.

\section{EARLY POSTNATAL MATERNAL DEPRESSIVE SYMPTOMS MAY PREDICT BEHAVIOURAL PROBLEMS AND AUTISM SYMPTOMS IN TODDLERS}

Ira Kleine, George Vamvakas, Alexandra Lautarescu, Andrew Pickles, David Edwards, Chiara Nosarti. UK

\subsection{6/bmjpo-2021-RCPCH.78}

Background Maternal depression has been studied as a risk factor for child development and found to be associated with less secure attachment, worse cognitive and behavioural outcomes in childhood, and a possible increased risk of autism spectrum disorder (ASD). Despite the wealth of research investigating postnatal parental depression, most studies have small sample sizes, assess parental mood during later infancy, use a dichotomous measure of parental depression, or examine distant child behavioural and emotional outcomes, with limited focus on ASD.

Objectives This study examined the association between early maternal postnatal depressive symptoms and offspring's mental health in a large cohort of term and preterm toddlers.

Methods Participants were 509 children enrolled in the Developing Human Connectome Project; 412 (80.9\%) were born at term, and $97(19.1 \%)$ were born preterm (<37 weeks gestation). Maternal postnatal depressive symptoms were assessed with the Edinburgh Postnatal Depression Scale (EPDS) at term. Children were followed-up at a median corrected age of 18.4 months (range 17.3 - 24.3) for neurodevelopmental assessment. Primary outcome measures were toddlers' Child Behaviour Checklist $1^{1 / 2}-5$ Total (CBCL) and Quantitative Checklist for Autism in Toddlers (Q-CHAT) scores. Secondary outcome measures were the CBCL internalising and externalising sub-scores. Cognition was assessed with the Bayley Scales of Infant and Toddler Development - Third Edition (BayleyIII). Multiple imputation $(n=40)$ was carried out to account for missing data. Data were analysed with multiple linear regression, including clinical and socio-demographic confounders.

Results Higher maternal EPDS scores were associated with toddlers' higher CBCL total scores $(B=0.93$, 95\% CI 0.43 $\left.1.44, \mathrm{p}<0.001, \mathrm{f}^{2}=0.05\right)$ and higher $\mathrm{Q}$-CHAT scores $(\mathrm{B}=0.27$, $95 \%$ CI $\left.0.03-0.52, \mathrm{p}<0.05, \mathrm{f}^{2}=0.01\right)$. Higher maternal EPDS 